\title{
Self-Assembled Monolayer of Mixed Gold and Nickel Nanoparticles
}

\author{
Yanni Jie ${ }^{1,2}$, Huiqing Fan $^{1 *}$, Wei You ${ }^{2 *}$
}

(Received 9 July 2012; accepted 19 September 2012; published online 25 September 2012.)

\begin{abstract}
Forming a monolayer of mixed nickel and gold nanoparticles through self-assembly via simple solution processing constitutes an important step toward inexpensive nanoparticle-based carbon nanofiber growth. In this work, mixed gold and nickel nanoparticles were anchored on the silicon wafer using self-assembled monolayers (SAMs) as a template. SAMs of 3-mercaptopropyl trimethoxysilane (MPTS-SAMs) were formed on silicon wafer, with the exposed thiol functionality providing ligand exchange sites to form the mixed monolayer of nickel and gold nanoparticles via a two-step sequential soaking approach. The densities of the nickel and gold nanoparticles on the surface can be varied by adjusting the soaking sequence.
\end{abstract}

Keywords: Nanoparticles; Self-assembled monolayer; 3-mercaptopropyl trimethoxysilane; Solution processing

Citation: Yanni Jie, Huiqing Fan and Wei You, "Self-Assembled Monolayer of Mixed Gold and Nickel Nanoparticles", Nano-Micro Lett. 4 (3), 166-171 (2012). http://dx.doi.org/10.3786/nml.v4i3.p166-171

\section{Introduction}

The nickel foils have been used to grow carbon microstructures since early 70s [1]. Previous studies of the carbon nanofiber growth process from nickel nanoparticles have demonstrated that nickel nanoparticles (5 $\mathrm{nm}-20 \mathrm{~nm}$ in diameter) can be successfully applied to the nanofiber growth [2]. Ab initio molecular dynamics simulations of the early stages of single-walled carbon nanotube (SWCNT) growth on gold nanoparticles showed that the root growth was not observed on gold. The reason lied in the fact that graphene adhesion energy is negligible (compared to $k T$ at $1200 \mathrm{~K}$ ) on gold surface while covalent bond formation requires significantly higher activation energy. Consequently, $\mathrm{C}$ atoms are not bound to the $\mathrm{Au}$ particle with sufficient time to allow for defect annealing and tube formation [3-5]. Compared with that of gold nanoparticles, the mixed nickel and gold nanoparticles have different activity towards the $\mathrm{C}$ atoms during the growth of the carbon nan- otube. In this paper, we describe a technique to form the mixed monolayer of nickel and gold nanoparticles on the surface of silicon wafer through self-assembly. As the gold nanoparticles constitute the vast majority of the particles, the nickel nanoparticles are spaced apart from each other. Nickel nanoparticles can be used as the catalyst to grow the carbon nanofiber. The goal of this paper is to study how to form the spaced nickel nanoparticles through self-assembly and simple solution processing, which constitutes an important step toward inexpensive nanoparticle-based carbon nanofiber growth.

\section{Experimental section}

\section{Nanoparticle synthesis}

All nanoparticles were synthesized via standard procedures with commercially available reagents. No further purification was conducted. The synthetic meth-

\footnotetext{
${ }^{1}$ State Key Laboratory of Solidification Processing, School of Materials Science, Northwestern Polytechnical University, Xi'an 710072, China

${ }^{2}$ Department of Chemistry, University of North Carolina at Chapel Hill, Chapel Hill, NC 27599-3290, USA

*Corresponding author. E-mail: hqfan3@163.com, wyou@email.unc.edu
} 
ods are based on previously reported procedures.

\section{Gold nanoparticles synthesis}

Au Nanoparticles were synthesized under ambient atmosphere by first bringing a solution of $1.45 \mathrm{ml}(4.41$ mmol) oleylamine (97\%, Pfaltz \& Bauer) in $25 \mathrm{ml}$ of toluene to reflux, followed by the fast injection of a solution of $0.60 \mathrm{ml}(1.82 \mathrm{mmol})$ oleylamine, $0.0863 \mathrm{~g}(0.219$ mmol) of $\mathrm{HAuCl}_{4} \cdot 3 \mathrm{H}_{2} \mathrm{O}(99.99 \%$, Alfa Aesar), and 1.0 $\mathrm{ml}$ toluene. The mixture was heated at $110^{\circ} \mathrm{C}$ for two hours before cooling to room temperature [6].

\section{Nickel nanoparticles synthesis}

Nickel nanoparticles were prepared by the thermaldriven reduction of $0.20 \mathrm{~g}(0.78 \mathrm{mmol}) \mathrm{Ni}(\mathrm{acac})_{2}(98 \%$, TCI America) in a mixture of $2.0 \mathrm{ml}(6.1 \mathrm{mmol})$ oleylamine and $5.0 \mathrm{~g}(11.6 \mathrm{mmol})$ of trioctylphosphine oxide (TOPO, 90\%, Sigma-Aldrich) in a $100 \mathrm{ml}$ threenecked, round-bottomed flask. After degassing under vacuum at $60^{\circ} \mathrm{C}$ for $2 \mathrm{~h}$ and backfilling with nitrogen, the solution was heated to $240^{\circ} \mathrm{C}$ and held at this temperature for $30 \mathrm{~min}$. As the temperature was increasing, when the temperature reached $100^{\circ} \mathrm{C}, 0.3 \mathrm{ml}$ trioctylphosphine (TOP, $97 \%$, Strem) was added by syringe. After heating, the mixture was cooled to room temperature and $\sim 5 \mathrm{ml}$ toluene was added to prevent crystallization of the TOPO [7].

The properties of the $\mathrm{Au}$ and $\mathrm{Ni}$ nanoparticles are shown in Table 1. The TEM images of the $\mathrm{Ni}$ and $\mathrm{Au}$ nanoparticles are dislayed in Fig. 1(a) and 1(b).

Table 1 Properties of $\mathrm{Au}$ and Ni nanoparticles

\begin{tabular}{ccc}
\hline & $\mathrm{Ni}$ & $\mathrm{Au}$ \\
\hline Solvent & Hexanes & Hexanes \\
Concentration & $1.3 \mathrm{mg} / \mathrm{ml}$ & $0.05 \mathrm{mg} / \mathrm{ml}$ \\
Size & $8 \sim 15 \mathrm{~nm}$ & $5 \sim 25 \mathrm{~nm}$ \\
Ligands & Oleic acid and TOP & Oleyamine \\
\hline
\end{tabular}

\section{Materials characterization}

Transmission electron microscopy (TEM) was performed with a JEOL 2000FX microscope operated at $200 \mathrm{kV}$. Scanning electron micrographs (SEM) and Energy-dispersive X-ray spectrographs (EDS) were obtained using the electron beam capabilities on a FEI Helios 600 Nanolab Dual Beam System (accelerating voltage $15 \mathrm{kV}$, beam current $20 \mathrm{nA}$ ).

\section{Preparation of 3-mercaptopropyl trimethoxysi- lane self-assembled monolayers (MPTS-SAMs) on silicon}

\section{Cleaning the silicon wafer}

The Si substrates were cleaned with the Radio Corporation of America (RCA) procedure. The RCA solution contained deionised water, ammonium hydroxide and hydrogen peroxide with volume ratio of $\mathrm{H}_{2} \mathrm{O}: \mathrm{NH}_{4} \mathrm{OH}: \mathrm{H}_{2} \mathrm{O}_{2}=2: 1: 1$. The silicon substrate was
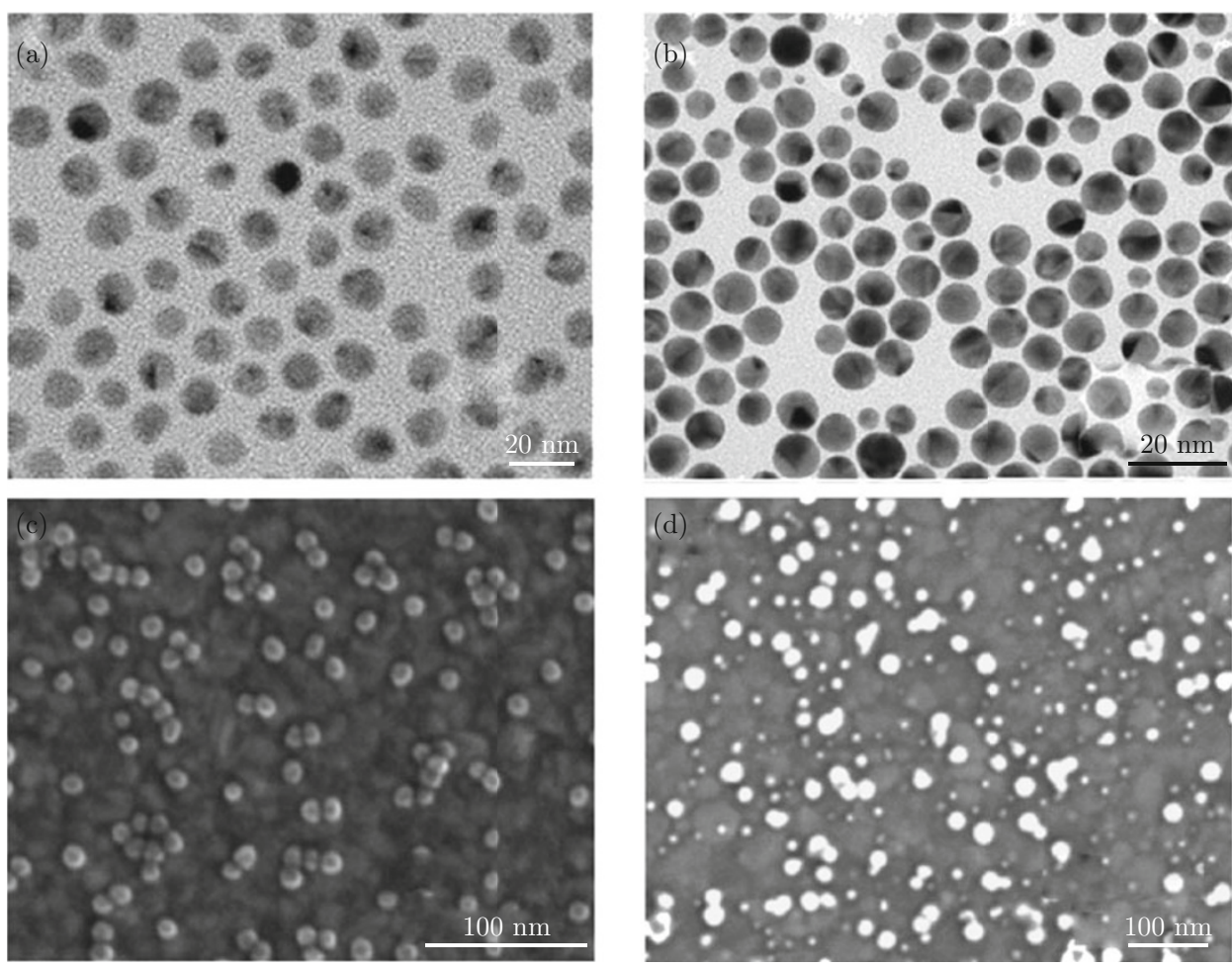

Fig. 1 TEM images of Nanoparticles. (a) Ni; (b) Au; SEM images of Nanoparticles on the Au substrate; (c) Ni; (d) Au. 
soaked in the RCA solution to remove the dust and contaminants, followed by exposure to UV/Ozone for $15 \mathrm{~min}$ to remove any organic residues. The substrates were then rinsed thoroughly with water and ethanol $(100 \%)$, dried with a stream of $\mathrm{N}_{2}$, and treated with ultraviolet light and ozone (UVO) for $20 \mathrm{~min}$ to remove any additional organic remaining.

\section{Forming MPTS-SAMs on silicon}

In this paper, a monolayer of MPTS with -SH functional group was assembled onto the silicon substrates (Fig. 2(b)). The preparation of the SAMs was performed according to literature [8]. N-type single-crystal silicon wafers $\{111\}$ with one side polished were used as the substrates after being cleaned as described above. A solution containing $5 \%$ silane, $95 \%$ hexanes and $0.1 \%$ acetic acid with respect to silane was prepared, with acetic acid as the catalyst. The as-cleaned silicon substrate was soaked in the solution for at least 4 hours.
The samples were thoroughly washed with EtOH after being taken out from the solution, and then put in tetrahydrofuran (THF) where physically absorbed molecules were removed by 1 min sonication. The samples were then rinsed with EtOH again and dried with $\mathrm{N}_{2}$. Fig. 2(a) and 2(b) summarize the procedure of preparing MPTS monolayer on the silicon wafer, which results in the thiol (-SH) functional groups on the surface of the silicon wafer.

\section{Formation of the nanoparticles monolayer on the MPTS-SAMs}

As functionalized wafer was then submerged into the nanoparticle solution. The Au nanoparticles with oleyamine ligands and $\mathrm{Ni}$ nanoparticles with oleic acid and TOP ligands can be covalently attached to the organic molecular monolayer surface functionalized with thiol (-SH) (MPTS-SAMs) via the ligand exchange [9], (a) $\mathrm{Si}$

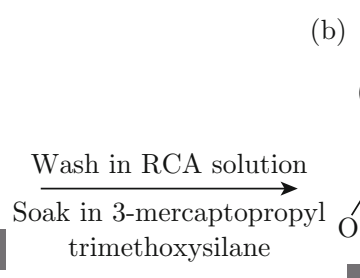

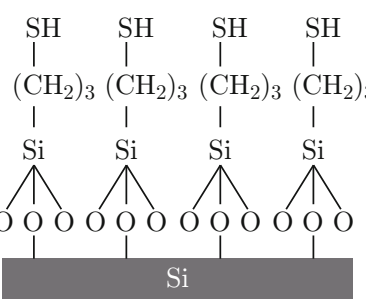

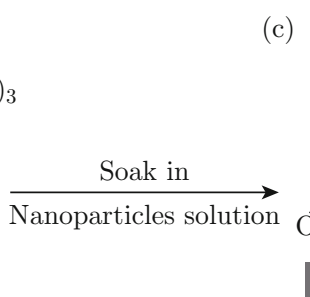

(c)

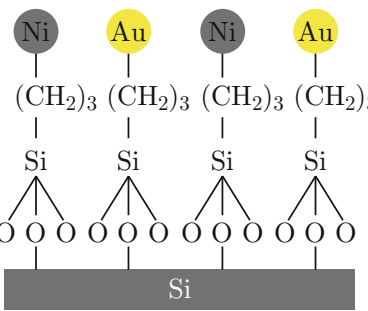

Fig. 2 The whole procedure of the experiment. (a) Bare Si surface; (b) After forming the 3-mercaptopropyl trimethoxysilane self-assembled monolayer on silicon surface; (c) After soaking in the Au and Ni nanoparticle solution.
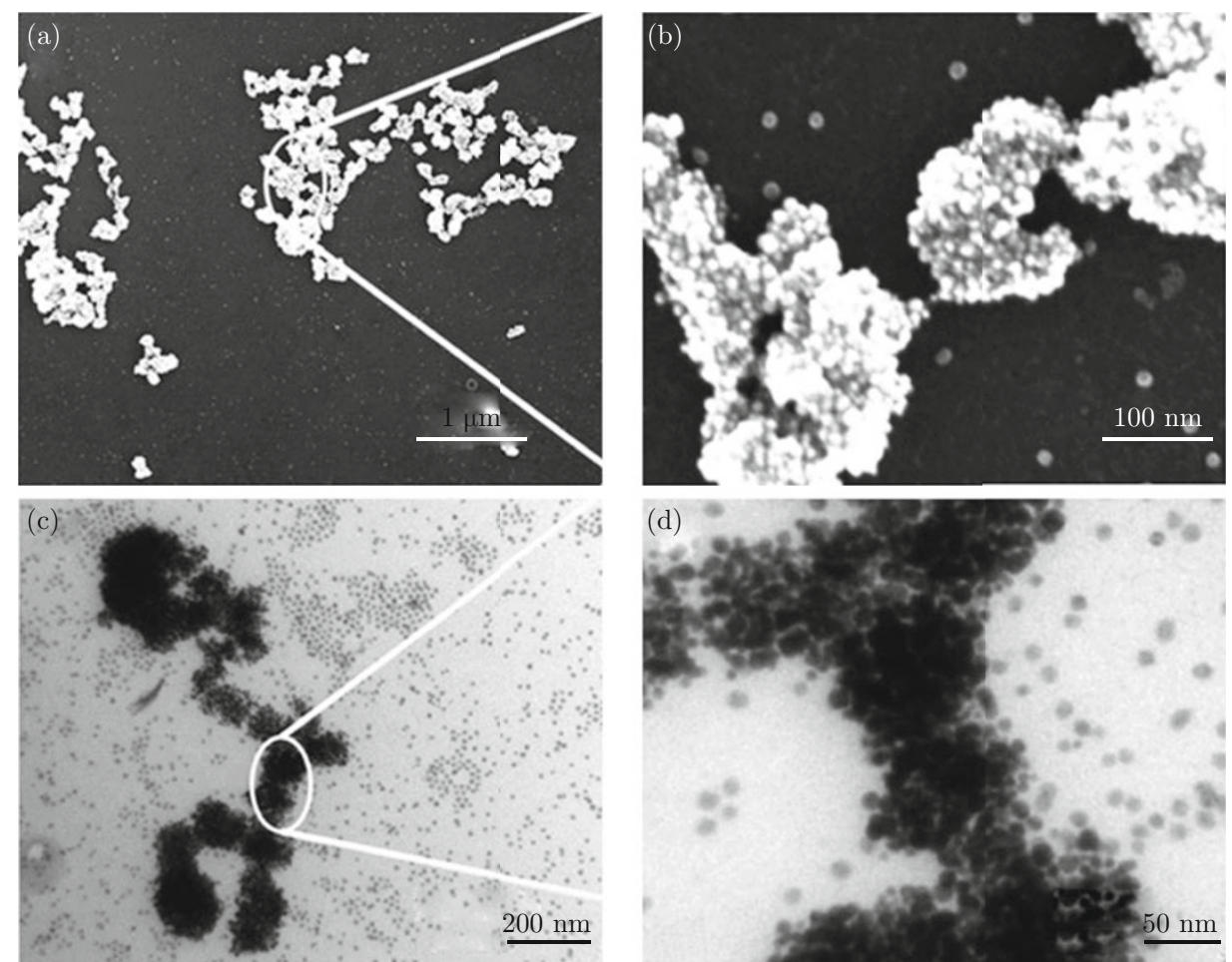

Fig. 3 (a) SEM image of the mixed clusters Ni and Au nanoparticles on silicon. (b) Zoom in (a). (c) TEM image of the clusters of mixed $\mathrm{Ni}$ and Au nanoparticles in solution. (d) Zoom in (c). 
as shown in Fig. 2(c). Various methods were attempted to form the mixed nanoparticles monolayers on the surface of the silicon wafer, as elaborated below.

\section{Via the solution of mixed nanoparticles}

Initially we tried to form the $\mathrm{Ni}$ and $\mathrm{Au}$ nanoparticle mixed monolayer by soaking the substrate in the solution of mixed nanoparticles, where the ratio of $\mathrm{Au}$ and Ni nanoparticles was 1:1. The silicon substrate with MPTS-SAMs was soaked in such solution of mixed nanoparticles for $60 \mathrm{~min}$. The results are shown in the Fig. 3.

\section{Soaked first in the $\mathrm{Ni}$ nanoparticles solution then in $\mathrm{Au}$}

We then tried to soak the MPTS-SAMs functionalized silicon wafer in a solution with only Ni nanoparticles for $60 \mathrm{~min}$. Then the supposedly Ni nanoparticles decorated Si wafer was washed with ethanol (200 proof), and dried with $\mathrm{N}_{2}$ stream. After this cleaning procedure, the same substrate was submerged in a solution with only $\mathrm{Au}$ nanoparticles for $10 \mathrm{~min}$. Immediately following the deposition, the substrate was sonicated in hexanes and then in THF for $30 \mathrm{~s}$ each. The nanoparticles remained on the substrate surface, implying the existence of chemical attachment had occurred. Moreover, the nanoparticles remained on the surface after attempting to remove them using adhesive tape. Fig. 4 shows the representative SEM image after such practice. Thus we believed that chemical attach- ment of these nanoparticles to the substrate via ligand exchange is likely the mechanism of forming monolymer of mixed nanoparticles.

\section{Soaked first in the $\mathrm{Au}$ nanoparticles solution then in $\mathrm{Ni}$}

Following a similar procedure as described in 2.4.2, we attempted to form the monolymer of mixed nanoparticles with a reversed soaking sequences, i.e. first in the solution with only $\mathrm{Au}$ nanoparticles for 10 min and then in the solution with only Ni nanoparticles for $60 \mathrm{~min}$. The SEM images of the nanoparticles on the substrate are shown in the Fig. 5.

\section{Results and discussion}

When the silicon substrate decorated with the SAMs of exposed thiol was soaked in the solution with mixed $\mathrm{Ni}$ and $\mathrm{Au}$ nanoparticles, the nanoparticles aggregated on the SAMs (Fig. 3(a) and 3(b)). TEM images of the mixed nanoparticle solution in Fig. 3(c) and 3(d) indicate that the $\mathrm{Au}$ and $\mathrm{Ni}$ nanoparticles already aggregated in the solution, even before the soaking took place. This is the main reason why we only observed the aggregated $\mathrm{Au}$ and $\mathrm{Ni}$ nanoparticles on the substrate after our attempted formation of monolayer of mixed nanoparticles. Therefore, we concluded that it would be difficult to prepare the monolayer of mixed $\mathrm{Au}$ and Ni nanoparticles on the SAMs by soaking substrate in the solution containing pre-mixed nanoparticles.

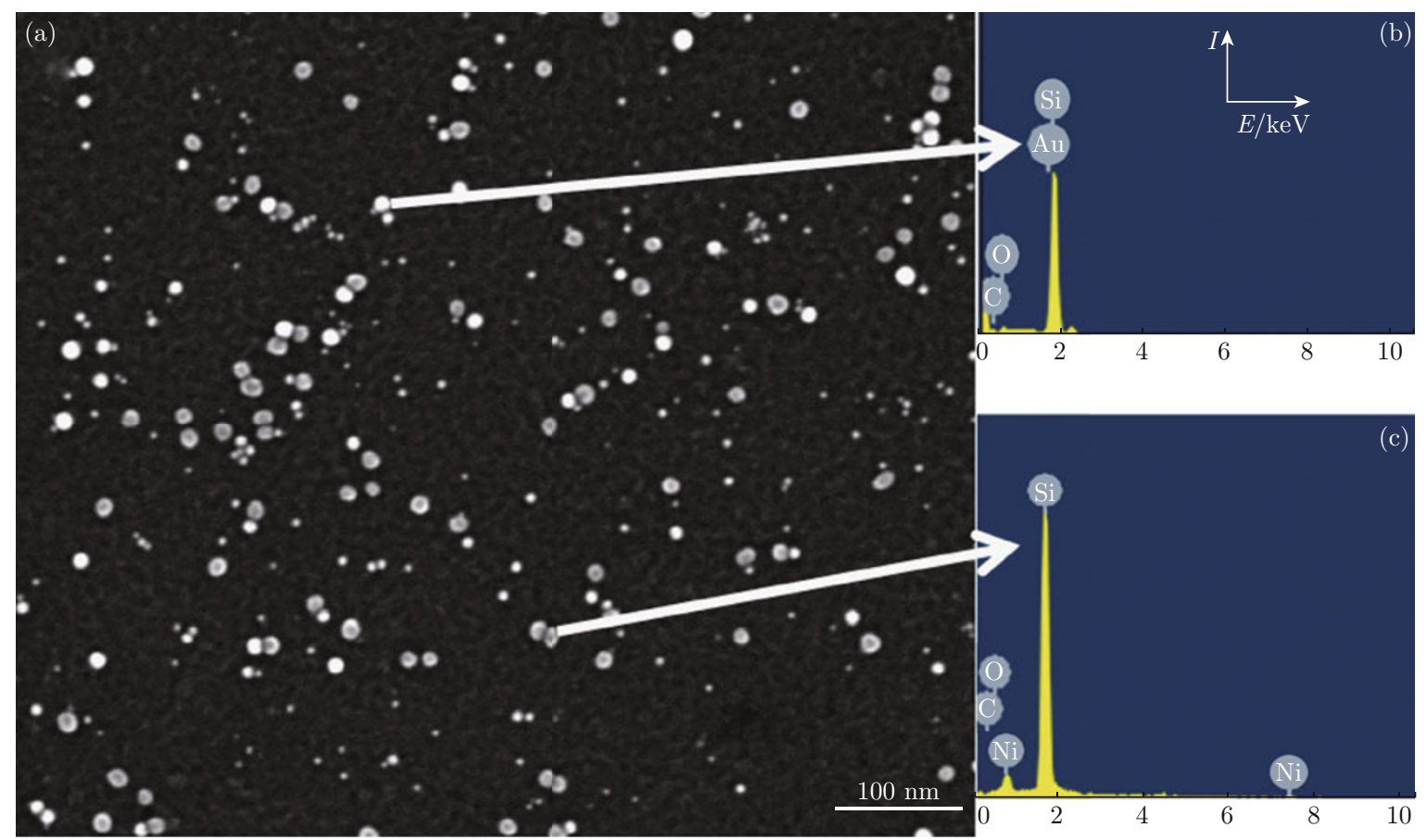

Fig. 4 (a) Mixed nanoparticles on the Silicon wafer. The sample was soaked first in the Ni nanoparticles solution then in $\mathrm{Au}$; (b) EDS on the Au nanoparticles; (c) EDS on the Ni nanoparticles. 


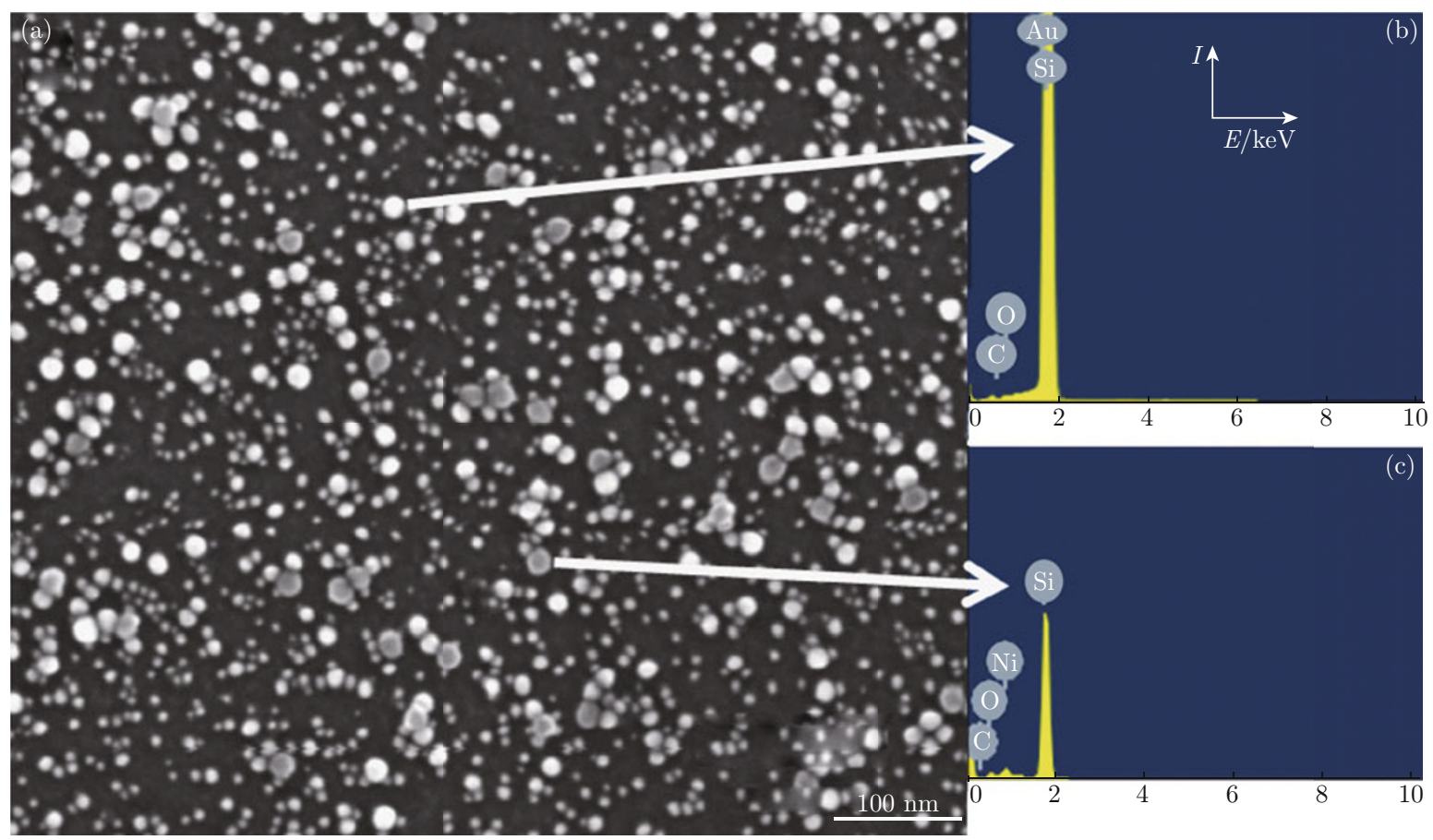

Fig. 5 (a) Mixed nanoparticles on the Silicon wafer. The sample was soaked first in the Au nanoparticles solution then in $\mathrm{Ni}$; (b) EDS on the Au nanoparticles; (c) EDS on the Ni nanoparticles.

However, if we take two steps, i.e., soaking the substrate in two individual solutions with only $\mathrm{Ni}$ or $\mathrm{Au}$ nanoparticles in sequence, we can achieve the desired monolayer of mixed $\mathrm{Ni}$ and $\mathrm{Au}$ nanoparticles on the SAMs (Fig. 4(a) and Fig. 5(a)). Subsequent analyses indicate that there are two different kinds of nanoparticles ( $\mathrm{Ni}$ and $\mathrm{Au}$ ) on silicon substrate. First, comparing the $\mathrm{Ni}$ and $\mathrm{Au}$ nanoparticles on the Au substrate (Fig. 1(c) and 1(d)), it is obvious that different nanoparticles have different contrast in the SEM images. The $\mathrm{Ni}$ nanoparticles appear gray while the $\mathrm{Au}$ nanoparticles appear brighter. Thus, it is relatively easy to distinguish between $\mathrm{Ni}$ and $\mathrm{Au}$ nanoparticles. Furthermore, these assignments (Ni or $\mathrm{Au}$ nanoparticles) were confirmed by the EDS, which detected the Ni signal on the gray particles and Au signal on the brighter particles.

Interestingly, the soaking sequence (i.e., into the solution of $\mathrm{Au}$ nanoparticles first, followed by the solution of $\mathrm{Ni}$ nanoparticles, or reserved order) can affect the density of nanoparticles on the substrate. When the monolayer of mixed nanoparticles are prepared with first soaking into the solution of $\mathrm{Ni}$ nanoparticles followed by the one of $\mathrm{Au}$ nanoparticles, we observe low densities of both the nanoparticles, with the density of the $\mathrm{Au}$ nanparticles even lower than that of the Ni nanoparticles. When we reserve the soaking sequence, densities of both the $\mathrm{Ni}$ and $\mathrm{Au}$ particles are higher. This observation may be ascribed to the relative reactivity of ligand exchange for dif- ferent nanoparticles towards the surface bound thiols [9]. For the nanofiber growth, a relatively higher $\mathrm{Au}$ density is preferred since the $\mathrm{Ni}$ nanoparticles can be spaced apart from each other by the vast amount of $\mathrm{Au}$ nanoparticles. As a result, individual nanofibers could be grown apart from each other since Ni nanoparticles can serve as the catalyst for such growth.

\section{Conclusion}

We use a very simple, reproducible and inexpensive method to form a monolayer with mixed $\mathrm{Ni}$ and $\mathrm{Au}$ nanoparticles. We can vary the density of $\mathrm{Ni}$ and $\mathrm{Au}$ particles by changing the soaking sequence of the solution containing individual nanoparticles. The Ni nanoparticles are isolated from each other by the $\mathrm{Au}$ nanoparticles on the substrate. We believe that such mixed monolayer of $\mathrm{Ni}$ and $\mathrm{Au}$ nanoparticles can find potential application in the controlled growth of carbon nanofibers, where $\mathrm{Ni}$ nanoparticles can serve as the catalyst to catalyze the formation of the nanofiber.

\section{Acknowledgements}

Y. J. gratefully acknowledges a Fellowship from Chinese Scholarship Council. We thank Aaron C. Johnston-Peck for preparing the $\mathrm{Au}$ and Ni nanoparticles. 


\section{References}

[1] T. Baird, J. R. Fryer and B. Grant, Carbon 12, 591 (1974). http://dx.doi.org/10.1016/ 0008-6223(74) 90060-8

[2] P. M. Ajayan, Nature 427, 402 (2004). http://dx.doi. org/10.1038/427402a

[3] J. Y. Raty, F. Gygi and G. Galli, Phys. Rev. Lett. 95, 096103 (2005). http://dx.doi.org/10. 1103/PhysRevLett. 95.096103

[4] M. Long, J. Jiang, Y. Li, R. Cao, L. Zhang and W. Cai, Nano-Micro Lett. 3, 171 (2011). http://dx.doi. org/10.3786/nml.v3i3.p171-177

[5] A. Azadbakht and A. R. Abbasi, Nano-Micro Lett. 2, 296 (2010). http://dx.doi.org/10.3786/nml.v2i4. p296-305

[6] H. Hiramatsu and F. E. Osterloh, Chem. Mater. 16, 2509 (2004). http://dx.doi.org/10.1021/cm049532v

[7] A. C. Johnston-Peck, J. Wang and J. B. Tracy, ACS Nano 3, 1077 (2009). http://dx.doi.org/10.1021/ $\mathrm{nn} 900019 \mathrm{x}$

[8] D. Huang, Z. D. Xiao, J. H. Gu, N. P. Huang and C. W. Yuan, Thin Solid Films 305, 110 (1997). http:// dx.doi.org/10.1016/S0040-6090(97)00202-2

[9] Y. Jie, J. R. Niskala, A. C. Johnston-Peck, P. J. Krommenhoek, J. B. Tracy, H. Fan and W. You, J. Mater. Chem. 22, 1962 (2012). http://dx.doi.org/10.1039/ c1jm14612b 